\title{
Exploring the likelihood of $\mathrm{CP}$ violation in neutrino oscillations
}

\author{
Gulsheen Ahuja \\ Department of Physics, Centre of Advanced Study, P.U., Chandigarh, India. \\ Email: gulsheenahuja@yahoo.co.in
}

November 13, 2018

\begin{abstract}
In view of the latest $\mathrm{T} 2 \mathrm{~K}$ and MINOS observations regarding the mixing angle $s_{13}$, we have explored the possibility of existence of CP violation in the leptonic sector. Using hints from the construction of the ' $d b$ ' unitarity triangle in the quark sector, we have made an attempt to construct the ' $\nu_{1} . \nu_{3}$ ' leptonic unitarity triangle, suggesting a good possibility of having non zero $\mathrm{CP}$ violation.
\end{abstract}

\section{Introduction}

The recent T2K [1] and MINOS [2] observations regarding the mixing angle $s_{13}$ has given a big impetus to the sharpening of the implications of the neutrino oscillations, in particular the non zero value of angle $s_{13}$ implies the possibility of existence of CP violation in the leptonic sector. In the context of neutrino oscillation phenomenology, the last few years have seen impressive advances in fixing the neutrino mass and mixing parameters through solar [3]- 9], atmospheric [10], reactor (KamLAND [11], CHOOZ [12]) and accelerator (K2K [13], MINOS [2, 14]) neutrino experiments. Adopting the three neutrino framework, several authors [11, 15]-[17] have presented updated information regarding these parameters obtained by carrying out detailed global analyses. In particular, incorporating the above mentioned developments regarding the angle $s_{13}$, Fogli et al. [17] have carried out a global three neutrino oscillation analysis, yielding

$$
\begin{gathered}
\Delta m_{21}^{2}=7.58_{-0.26}^{+0.22} \times 10^{-5} \mathrm{eV}^{2}, \quad\left|\Delta \mathrm{m}_{31}^{2}\right|=2.35_{-0.21}^{+0.12} \times 10^{-3} \mathrm{eV}^{2} \\
\sin ^{2} \theta_{12}=0.312_{-0.016}^{+0.017}, \quad \sin ^{2} \theta_{23}=0.42_{-0.03}^{+0.08}, \quad \sin ^{2} \theta_{13}=0.025 \pm 0.007
\end{gathered}
$$

In analogy with the quark mixing phenomenon, the above value of $s_{13}$ which is not so 'small' suggests likelihood of CP violation in the leptonic sector. A comparison of the 
mixing angles in the leptonic sector with those in the quark sector point out that the $\mathrm{CP}$ violation could, in fact, be considerably large in this case. This possibility, in turn, can have deep phenomenological implications. As is well known, the two CP violating Majorana phases do not play any role in the case of neutrino oscillations, therefore any hint regarding the value of Dirac-like $\mathrm{CP}$ violating phase in the leptonic sector $\delta_{l}$ will go a long way in the formulation of proposals on observation of $\mathrm{CP}$ violation in the Long BaseLine (LBL) experiments [13, 14, 18. In the absence of any hints from the data regarding leptonic $\mathrm{CP}$ violation, keeping in mind the parallelism between the neutrino mixing and the quark mixing, an analysis of the quark mixing phenomena could provide some viable clues regarding this issue in the leptonic sector.

It may be noted that in the context of fermion mixing phenomena, the PontecorvoMaki-Nakagawa-Sakata (PMNS) [19, 20] and the Cabibbo-Kobayashi-Maskawa (CKM) [21, 22] matrices have similar parametric structure. Also, regarding the three mixing angles corresponding to the quark and leptonic sector, it is interesting to note that in both the cases the mixing angle $s_{13}$ is smaller as compared to the other two. Taking note of these similarities of features, in this paper, using the analogy of the quark mixing case we have made an attempt to find the possibility of the existence of $\mathrm{CP}$ violation in the leptonic sector.

The detailed plan of the paper is as follows. In Section (2), we examine the quark mixing case for obtaining useful hints for the case of lepton mixing, studied in Section(3). Finally, in Section (44) we summarize and conclude.

\section{Quark mixing case}

In this context, we first examine the case of quark mixing, wherein the CKM matrix as well as the existence of $\mathrm{CP}$ violation are well established. Parallel to the leptonic sector wherein only the three mixing angles or correspondingly the magnitudes of the three elements of the mixing matrix are known, one would like to consider a similar situation in the quark sector and examine whether one can deduce any viable information regarding the existence of $\mathrm{CP}$ violation in the quark mixing phenomena. In this context, several important features in the case of CKM paradigm having implications for $\mathrm{CP}$ violation may be kept in mind. In particular, one may note that the magnitudes of the CKM matrix elements are rephasing invariant implying that these are independent of the 36 parameterizations of the CKM matrix. Also one has to keep in mind that in any parameterization the $\mathrm{CP}$ violating phase of the CKM matrix can be related to the magnitudes of its elements. Therefore, in case one can find the magnitudes of the CKM matrix elements, even approximately, one may be able to get some idea about the $\mathrm{CP}$ violation. In this context, the Particle Data Group (PDG) representation [23] of the CKM matrix facilitates the approximate construction of the magnitudes of the CKM matrix elements from the three well known mixing angles or the elements $V_{u s}, V_{c b}$ and $V_{u b}$, with one of the angles being much smaller than the other two. The approximate magnitudes of the CKM matrix elements then provide a way to construct the unitarity triangles which 
can be used to determine the Jarlskog's rephasing invariant parameter $J$, an important parameter as all CP violating effects are proportional to it. Further, with the knowledge of $J$, the CP violating phase $\delta$ can be determined in the PDG representation. The same is true in the leptonic sector also.

To this end, we first construct the approximate magnitudes of the elements of the quark mixing matrix. For ready reference as well as to facilitate discussion of results, the Cabibbo-Kobayashi-Maskawa (CKM) matrix [21, 22] is defined as

$$
\left(\begin{array}{c}
d^{\prime} \\
s^{\prime} \\
b^{\prime}
\end{array}\right)=\left(\begin{array}{lll}
V_{u d} & V_{u s} & V_{u b} \\
V_{c d} & V_{c s} & V_{c b} \\
V_{t d} & V_{t s} & V_{t b}
\end{array}\right)\left(\begin{array}{c}
d \\
s \\
b
\end{array}\right)
$$

which in the PDG representation [23] involving angles $\theta_{12}, \theta_{23}, \theta_{13}$ and the phase $\delta$ is given as

$$
V_{C K M}=\left(\begin{array}{ccc}
c_{12} c_{13} & s_{12} c_{13} & s_{13} e^{-i \delta} \\
-s_{12} c_{23}-c_{12} s_{23} s_{13} e^{i \delta} & c_{12} c_{23}-s_{12} s_{23} s_{13} e^{i \delta} & s_{23} c_{13} \\
s_{12} s_{23}-c_{12} c_{23} s_{13} e^{i \delta} & -c_{12} s_{23}-s_{12} c_{23} s_{13} e^{i \delta} & c_{23} c_{13}
\end{array}\right)
$$

with $c_{i j}=\cos \theta_{i j}$ and $s_{i j}=\sin \theta_{i j}$ for $i, j=1,2,3$.

Making use of the fact the mixing angle $s_{13}\left(\equiv V_{u b}\right)$ is small in comparison to both $s_{12}\left(\equiv V_{u s}\right)$ and $s_{23}\left(\equiv V_{c b}\right)$ as well as using their values given by PDG 2010 [23] and the above representation of the CKM matrix, the approximate magnitudes of the CKM matrix elements come out to be

$$
V_{C K M}=\left(\begin{array}{ccc}
0.97431 \pm 0.00021 & 0.2252 \pm 0.0009 & 0.00389 \pm 0.00044 \\
0.2250 \pm 0.0009 & 0.97351 \pm 0.00021 & 0.0406 \pm 0.0013 \\
0.00914 \pm 0.00029 & 0.0396 \pm 0.0013 & 0.999168 \pm 0.000053
\end{array}\right)
$$

The above matrix is in fairly good agreement with the one given recently by PDG 2010 [23].

Making use of the above matrix, one can construct the usually considered ' $d b$ ' unitarity triangle in the quark sector, expressed through the relation

$$
V_{u d} V_{u b}{ }^{*}+V_{c d} V_{c b}{ }^{*}+V_{t d} V_{t b}{ }^{*}=0 .
$$

Further, assuming the existence of CP violation, the Jarlskog's rephasing invariant parameter $J$, equal to twice the area of the unitarity triangle, can then be easily constructed using the magnitudes of the elements of the CKM matrix. Consequently, in the PDG representation, the $\mathrm{CP}$ violating phase $\delta$ can also be evaluated by using the relation between the parameter $J$ and phase $\delta$, e.g., $J=s_{12} s_{23} s_{13} c_{12} c_{23} c_{13}^{2} \sin \delta$. Following the procedure underlined in [24], we have obtained a histogram of $J$, shown in figure 1a, from which one can find

$$
J=(3.36 \pm 0.38) \times 10^{-5}
$$




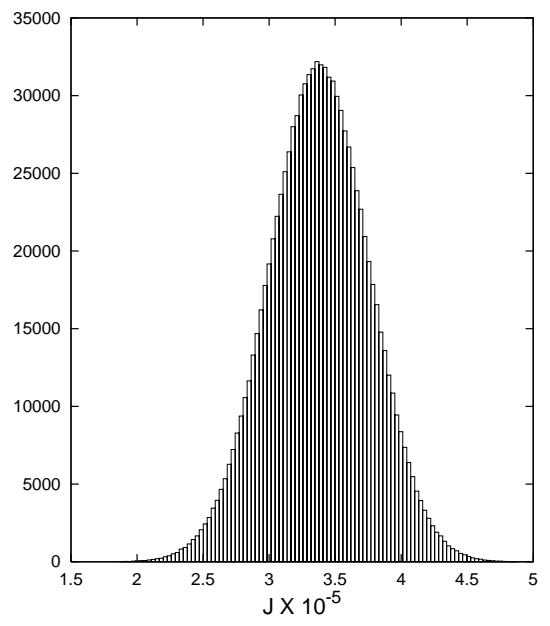

(a)

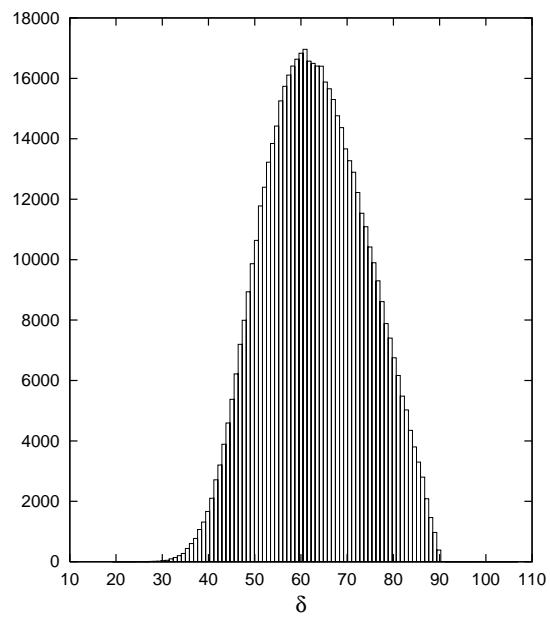

(b)

Figure 1: Histogram of $J$ and $\delta$ for ' $d b$ ' triangle in the case of quarks

the corresponding histogram of $\delta$, shown in figure 1 $\mathrm{b}$, yields

$$
\delta=62.60^{\circ} \pm 10.98^{\circ}
$$

Interestingly, we find that the above mentioned $J$ and $\delta$ values are compatible with those given by PDG 2010 [23].

\section{Lepton mixing case}

The above discussion as well as the present information regarding the neutrino mixing angle $s_{13}$ immediately provide a clue for exploring the possibility of existence of CP violation in the leptonic sector. We begin with the neutrino mixing matrix [19, 20],

$$
\left(\begin{array}{c}
\nu_{e} \\
\nu_{\mu} \\
\nu_{\tau}
\end{array}\right)=\left(\begin{array}{ccc}
U_{e 1} & U_{e 2} & U_{e 3} \\
U_{\mu 1} & U_{\mu 2} & U_{\mu 3} \\
U_{\tau 1} & U_{\tau 2} & U_{\tau 3}
\end{array}\right)\left(\begin{array}{c}
\nu_{1} \\
\nu_{2} \\
\nu_{3}
\end{array}\right)
$$

where $\nu_{e}, \nu_{\mu}, \nu_{\tau}$ are the flavor eigenstates and $\nu_{1}, \nu_{2}, \nu_{3}$ are the mass eigenstates. Following PDG representation, involving three angles and the Dirac-like CP violating phase $\delta_{l}$ as well as the two Majorana phases $\alpha_{1}, \alpha_{2}$, the PMNS matrix $U$ can be written as

$U=\left(\begin{array}{ccc}c_{12} c_{13} & s_{12} c_{13} & s_{13} e^{-i \delta_{l}} \\ -s_{12} c_{23}-c_{12} s_{23} s_{13} e^{i \delta_{l}} & c_{12} c_{23}-s_{12} s_{23} s_{13} e^{i \delta_{l}} & s_{23} c_{13} \\ s_{12} s_{23}-c_{12} c_{23} s_{13} e^{i \delta_{l}} & -c_{12} s_{23}-s_{12} c_{23} s_{13} e^{i \delta_{l}} & c_{23} c_{13}\end{array}\right)\left(\begin{array}{ccc}e^{i \alpha_{1} / 2} & 0 & 0 \\ 0 & e^{i \alpha_{2} / 2} & 0 \\ 0 & 0 & 1\end{array}\right)$

The Majorana phases $\alpha_{1}$ and $\alpha_{2}$ do not play any role in neutrino oscillations and henceforth would be dropped from the discussion. 


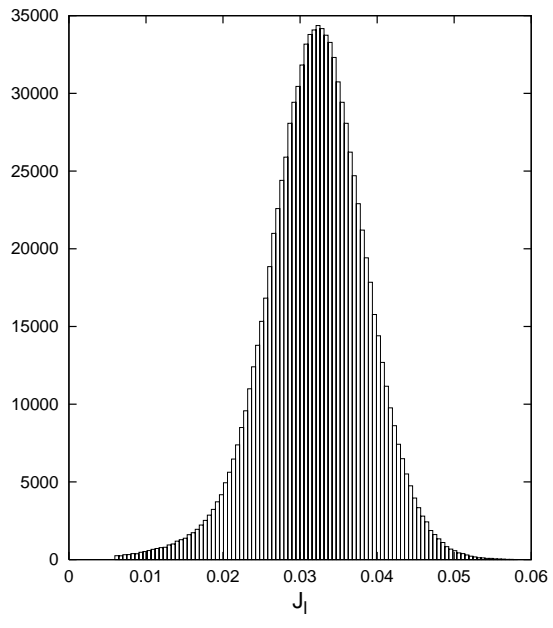

(a)

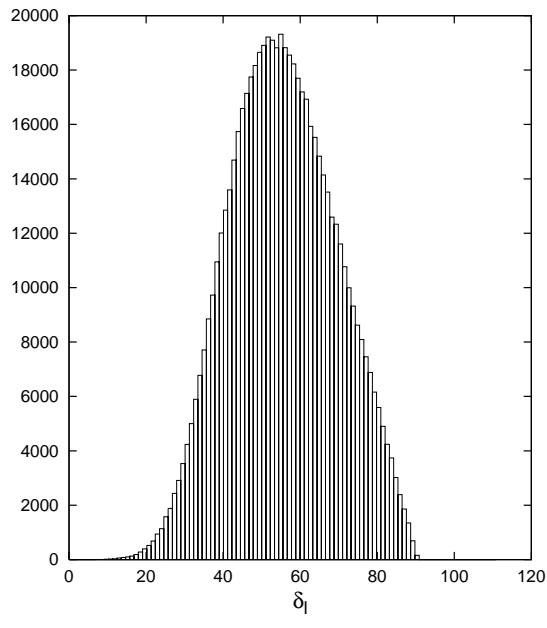

(b)

Figure 2: Histogram of $J_{l}$ and $\delta_{l}$ for ' $\nu_{1} . \nu_{3}$ ' triangle in the case of neutrinos

Before coming to the issue of existence of $\mathrm{CP}$ violation in the leptonic sector, we have to first determine the approximate magnitudes of the elements of the PMNS matrix. To this end, analogous to the construction of the CKM matrix presented in Eq. (5) and using the inputs given in Eq. (2), we get

$$
U=\left(\begin{array}{lll}
0.8190 \pm 0.0105 & 0.5516 \pm 0.0151 & 0.1581 \pm 0.0221 \\
0.4254 \pm 0.0315 & 0.6317 \pm 0.0442 & 0.6399 \pm 0.0610 \\
0.3620 \pm 0.0358 & 0.5376 \pm 0.0516 & 0.7520 \pm 0.0519
\end{array}\right)
$$

It is interesting to note that the present neutrino mixing matrix is compatible with those given by [25]-[28].

Analogous to the ' $d b$ ' triangle in the quark sector, we have considered the ' $\nu_{1} \cdot \nu_{3}$ ' unitarity triangle, expressed as

$$
U_{e 1} U_{e 3}^{*}+U_{\mu 1} U_{\mu 3}^{*}+U_{\tau 1} U_{\tau 3}^{*}=0 .
$$

Following the same procedure as in the quark case, using the matrix given in Eq. (11), we obtain the Jarlskog's rephasing invariant parameter in the leptonic sector $J_{l}$ as

$$
J_{l}=0.0318 \pm 0.0065
$$

corresponding distribution has been plotted in figure 2 a. It may be noted that in most of the recent analyses of neutrino oscillation phenomenology [29]-[31], it is usual to calculate the upper limit of $J_{l}$, however, in the present analysis we have calculated its likely range. Also, we find the corresponding phase $\delta_{l}$ from the histogram given in figure $2 \mathrm{~b}$ as

$$
\delta_{l}=54.98^{\circ} \pm 13.81^{\circ}
$$


Interestingly, this likely value of the phase $\delta_{l}$ is largely in agreement with several phenomenological analyses [32, 33]. Further, it is interesting to note that the present analysis carried out purely on phenomenological inputs is also very much in agreement with several analyses based on expected outputs from different experimental scenarios [34]-[36]. In particular, this value reinforces the conclusions of Marciano and Parsa [35] for the BNL-Homestake $(2540 \mathrm{~km})$ proposal.

\section{Summary and conclusions}

To summarize, in view of the latest T2K and MINOS observations regarding the mixing angle $s_{13}$ along with the other two well measured mixing angles $s_{12}$ and $s_{23}$ we have explored the possibility of existence of $\mathrm{CP}$ violation in the leptonic sector. Taking clues from the construction of the ' $d b$ ' unitarity triangle in the quark sector, we have made an attempt to construct the ' $\nu_{1} \cdot \nu_{3}$ ' leptonic unitarity triangle, suggesting a good possibility of having non zero $\mathrm{CP}$ violation. In particular, we find likely value of the $\mathrm{CP}$ violating phase in the leptonic sector to be $54.98^{\circ} \pm 13.81^{\circ}$.

\section{Acknowledgments}

The author would like to thank M. M. Gupta for suggesting the problem and for a careful reading of the manuscript. Thanks are also due to DST, Government of India for financial support and the Chairman, Department of Physics for providing facilities to work in the department.

\section{References}

[1] T2K Collab. (K. Abe et al.), Phys. Rev. Lett. 107, 041801 (2011), arXiv:1106.2822.

[2] MINOS Collab. (L. Whitehead), Recent results from MINOS, Joint ExperimentalTheoretical Seminar (24 June 2011, Fermilab, USA). Websites: theory.fnal.gov/jetp, http://www-numi.fnal.gov/pr plots/

[3] R. Davis, Prog. Part. Nucl. Phys. 32, 13 (1994).

[4] HOMESTAKE Collab. (B. T. Cleveland et al.), Astrophys. J. 496, 505 (1998).

[5] GALLEX Collab. (W. Hampel et al.), Phys. Lett. B 447, 127 (1999).

[6] GNO Collab. (M. Altmann et al.), Phys. Lett. B 616, 174 (2005), hep-ex/0504037.

[7] SUPER-KAMIOKANDE Collab. (J. P. Cravens et al.), Phys. Rev. D 78, 032002 (2008), arXiv:0803.4312. 
[8] SAGE Collab. (J. N. Abdurashitov et al.), Phys. Rev. C 80, 015807 (2009), arXiv:0901.2200.

[9] SNO Collab. (B. Aharmim et al.), Phys. Rev. C 81, 055504 (2010), arXiv:0910.2984.

[10] SUPER-KAMIOKANDE Collab. (R. Wendell et al.), Phys. Rev. D 81, 092004 (2010), arXiv:1002.3471.

[11] KamLAND Collab. (A. Gando et al.), Phys. Rev. D 83, 052002 (2011), arXiv:1009.4771.

[12] CHOOZ Collab. (M. Appolonio et al.), Eur. Phys. J. C 27, 331 (2003), hep-ex/0301017.

[13] K2K Collab. (M. H. Ahn et al.), Phys. Rev. D 74, 072003 (2006), hep-ex/0606032.

[14] MINOS Collab. (A. Habig) Mod. Phys. Lett. A 25, 1219 (2010), arXiv:1004.2647.

[15] T. Schwetz, M. Tortola and J. W. F. Valle, New J. Phys. 10, 113011 (2008), arXiv:0808.2016.

[16] G. L. Fogli, E. Lisi, A. Marrone, A. Palazzo and A. M. Rotunno, Phys. Rev. Lett. 101, 141801 (2008), arXiv:0806.2649.

[17] G. L. Fogli, E. Lisi, A. Marrone, A. Palazzo and A. M. Rotunno, arXiv:1106.6028.

[18] OPERA Collab. (N. Agafonova et al.), Phys. Lett. B 691, 138 (2010), arXiv:1006.1623.

[19] B. Pontecorvo, Zh. Eksp. Teor. Fiz. (JETP) 33, 549 (1957); ibid. 34, 247 (1958); ibid. 53, 1717 (1967).

[20] Z. Maki, M. Nakagawa and S. Sakata, Prog. Theor. Phys. 28, 870 (1962).

[21] N. Cabibbo, Phys. Rev. Lett. 10, 531 (1963).

[22] M. Kobayashi and T. Maskawa, Prog. Theor. Phys. 49, 652 (1973).

[23] K. Nakamura et al., JPG 37, 075021 (2010), updated results available at http://pdg.lbl.gov/.

[24] M. Randhawa and M. Gupta, Phys. Lett. B 516, 446 (2001), hep-ph/0106161.

[25] A. Strumia and F. Vissani, hep-ph/0606054.

[26] C. Giunti, Nucl. Phys. Proc. Suppl. 169, 309 (2007), hep-ph/0611125.

[27] M. C. Gonzalez-Garcia and M. Maltoni, Phys. Rept. 460, 1 (2008), arXiv:0704.1800.

[28] G. C. Branco and J. I. Silva-Marcos, Phys. Rev. D 80, 073016 (2009), arXiv:0907.121. 
[29] M. Obara and Z. Z. Xing, Phys. Lett. B 644, 136 (2007), hep-ph/0608280.

[30] H. Nunokawa, S. Parke and J. W. F. Valle, Prog. Part. Nucl. Phys. 60, 338 (2008), arXiv:0710.0554.

[31] R. Mohanta and D. John, Mod. Phys. Lett. A 26, 205 (2011), arXiv:1010.0288.

[32] Y. Farzan and A. Yu. Smirnov, Phys. Rev. D 65, 113001 (2002), hep-ph/0201105.

[33] K. R. S. Balaji, G. Couture and C. Hamzaoui, Phys. Rev. D 74, 033013 (2006), hep-ph/0605066.

[34] R. N. Mohapatra et al., Rept. Prog. Phys. 70, 1757 (2007), hep-ph/0510213.

[35] W. Marciano and Z. Parsa, hep-ph/0610258.

[36] H. Sugiyama, hep-ph/0411209. 